\title{
Manual Didáctico En Braille Para El Aprendizaje De Inglés Básico De Los Estudiantes No Videntes De La Asociación Aprodvich De La Ciudad De Riobamba
}

\author{
Luis Oswaldo Guadalupe Bravo \\ Marco Antonio Bravo Montenegro \\ Alberto Fabián Altamirano Pazmiño \\ Enrique Jesús Guambo Yerovi \\ Gabriel Isaac Orozco Yanez
}

Docente - Investigador del Centro de Idiomas

ESCUELA SUPERIOR POLITÉCNICA DE CHIMBORAZO - ECUADOR

Marcelo Eduardo Allauca Peñafiel

Técnico del Centro de Idiomas

ESCUELA SUPERIOR POLITÉCNICA DE CHIMBORAZO - ECUADOR

Carla Sofía Arguello Guadalupe

Docente - Investigador de la Facultad de Recursos Naturales

UNIVERSIDAD NACIONAL DE CHIMBORAZO - ECUADOR

doi: 10.19044/esj.2017.v13n22p59 URL:http://dx.doi.org/10.19044/esj.2017.v13n22p59

\begin{abstract}
The objective of the present research is the elaboration and application of a didactic manual with communicative strategies to develop Basic English for students of the Provincial Association of the Visually Impaired of Chimborazo (APRODVICH). The didactic manual seeks to provide techniques and instruments that support the Teaching Learning process of a second language according to the specific needs of the group under study. It becomes a progressive and systematic teaching instrument consisting of six units that include vocabulary activities, basic structures and use of questions that were applied in the classroom with the accompaniment of the teacher. The research has a quasi-experimental, correlational, descriptive, explanatory, applicative, field and bibliographic design. The technique of direct observation was used with the application of checklists and questionnaires reviewed and endorsed by academic peers that allowed the evaluation of the knowledge acquired in students such as spelling, pronunciation and comprehension. The sample was evaluated in two instances - pre and post - and the results were tabulated, analyzed, interpreted and contrasted which determined the progress and significant
\end{abstract}


development in pronunciation, use of the verb structure To-Be and whquestions of the English language in blind students.

Keywords: Manual, didactic, communicative strategies, aprodvich

\section{Resumen}

El objetivo de la presente investigación es la elaboración y aplicación de un manual didáctico con estrategias comunicativas para el desarrollo del inglés básico en los estudiantes de la Asociación Provincial de Discapacitados Visuales de Chimborazo (APRODVICH). El manual didáctico busca proporcionar técnicas e instrumentos que apoyen el proceso de enseñanza aprendizaje del idioma inglés, acordes a las necesidades específicas del grupo en estudio. Se convierte en un instrumento de enseñanza progresiva y sistemática, que cuenta con seis unidades, con actividades de vocabulario, estructuras básicas y uso de preguntas, que fueron aplicadas en el aula con el acompañamiento del docente. La investigación tiene un diseño cuasi experimental, correlacional, descriptivo, explicativo, aplicativo, de campo y bibliográfico. Se utilizó la técnica de observación directa con la aplicación de listas de cotejo y cuestionarios revisados y avalados por pares académicos que permitieron evaluar el conocimiento adquirido en los estudiantes como: El deletreo, la pronunciación y comprensión. La muestra fue evaluada en dos momentos (pre y post), y los resultados fueron tabulados, analizados, interpretados $\mathrm{y}$ contrastados, mismos que determinaron el avance y desenvolvimiento significativo en la pronunciación, uso de la estructura del verbo To-Be y "wh-questions" del idioma en los estudiantes no videntes.

Palabras-Clave: Manual, Didáctico, Estrategias Comunicativas, Aprodvich

\section{Introducción}

La importancia de las estrategias comunicativas en "El proceso enseñanza aprendizaje del habla Inglés básico con los estudiantes no videntes requieren de un proceso didáctico, intenso y práctico, que conlleve a nuevas experiencias y asimilación de conocimientos hacia una comunicación activa a través del diálogo" (Castañeda, 2009:98).

"El manual en el proceso educativo es un aporte fundamental, porque posee una serie de estrategias y actividades prácticas que realizan los estudiantes con la orientación de los docentes en función de los contenidos establecidos en el currículum" (Martínez Bonafé, 2002:64)

Es por esto que el manual didáctico con estrategias comunicativas, permite a los estudiantes de la Asociación Provincial de Discapacitados Visuales de Chimborazo (APRODVICH) desarrollar progresivamente la 
habilidad de hablar "Inglés Básico". Basado en el Marco Común Europeo, el proceso de enseñanza-aprendizaje que provee el manual facilita al estudiante ciego el aprendizaje de otro idioma. Además no se descarta implementar estrategias activas y prácticas que conduzcan a mejorar su patrimonio lingüístico, para lo cual el profesor de inglés debe implementar instrumentos didácticos en la enseñanza práctica y los recursos tecnológicos para dirigir a los estudiantes ciegos en el desarrollo de la comprensión oral del inglés. "Aprender el vocabulario en Inglés es muy importante, en virtud de que su estructura se utiliza en todas las conversaciones diarias. Para lo cual requiere específicamente dominar la gramática y el vocabulario para poder hablar el idioma con fluidez" (Owens \& E., 1999:45).

Una de las estructuras básicas utiliza el verbo "to be" y las otras cinco estructuras de oración en el idioma inglés, basadas en el Marco Común Europeo para que los estudiantes puedan identificarse y expresarse en presente, pasado, futuro y poder escuchar la oración en inglés y traducirla al español o viceversa, esto ayudará a los estudiantes ciegos a interrelacionar con sus compañeros. Por otro lado, el uso de preguntas- wh permiten a los estudiantes comunicarse y realizar preguntas básicas de ubicación, características de personas y demás temas importantes que le permiten relacionar con otros en un dialogo usando el idioma. "El proceso pedagógico es un sistema de comunicación cuya meta, de manera general, es modificar por repetición los actos de información, el repertorio de signos, rutinas, técnicas y formas de comportamiento, que se encuentran a disposición del receptor (educación pasiva) o del emisor (educación activa)" (Moles \& Zeltman, 2003:72).

De ahí que el desarrollo de instrumentos comunicativos ocupan un lugar estratégico en el conocimiento y aprendizaje del habla del inglés en estudiantes ciegos porque están en la capacidad de aprender a través de lo que oyen y tocan utilizando material didáctico apropiado para estas personas con necesidades especiales. "La comunicación es el proceso de codificación y descodificación de signos lingüísticos que requiere el intercambio de mensajes. Comunicación también significa poder, y el poder democrático se adquiere, en gran parte, por la capacidad comunicativa. El proceso didáctico se puede entender como un proceso comunicativo que tiene en cuenta las reglas y códigos del sistema de comunicación" (Gispert, 2006:36).

El problema del aprendizaje y desenvolvimiento lingüístico del inglés, en el Ecuador, resulta un factor crítico puesto que no existe interés, en la sociedad en general, por aprender y practicar un nuevo idioma. Sumado a esto, el aprendizaje de los estudiantes no videntes es más complejo porque los docentes no cuentan con instrumentos de aprendizaje efectivos y adaptables a ésta condición. Por esta razón la investigación trasciende a los contenidos básicos de la enseñanza del idioma Inglés, considerando también 
los procesos que promueven la participación activa en función de estrategias comunicativas que permiten alcanzar los aprendizajes esperados. Se procuran nuevos aprendizajes, mediante el desarrollo de destrezas al escuchar y hablar, que permitan al estudiante no vidente describir las imágenes que desarrolla en su mente de acuerdo a su realidad y condiciones específicas.

El aprendizaje del idioma ingles para la mayoría de personas resulta difícil, mucho más para los estudiantes no videntes, quienes deben superar esta barrera y desarrollar más sus otros sentidos. Razón por la cual es importante invitar a buscar alternativas prácticas para poder motivar e incentivar a los estudiantes a alcanzar conocimientos básicos de la lengua del Inglés, por esto surge la necesidad de crear un manual con estrategias comunicativas denominado LGB (Luis Guadalupe Bravo), con el objetivo principal de mejorar la destreza del speaking (hablar) y del listening (escuchar) de los estudiantes no videntes.

Al crearse la necesidad de generar procesos de enseñanza aprendizaje del inglés de manera activa, participativa y práctica es pertinente la aplicabilidad del manual LGB para que se constituya en calidad de herramienta pedagógica importante para trabajar de manera exclusiva con los estudiantes no videntes, haciendo uso de las destrezas para hablar y escuchar, ya que es el medio más directo para que puedan asimilar de mejor forma y por ende desenvolverse en su entorno o sociedad en general.

"El proceso pedagógico es un sistema de comunicación cuya meta, de manera general, es modificar por repetición los actos de información, el repertorio de signos, rutinas, técnicas y formas de comportamiento, que se encuentran a disposición del receptor o del emisor." (Moles \& Zeltman, 2013: 15)

Frente a la problemática existente con los estudiantes no videntes de la Asociación APRODVICH se plantea estrategias comunicativas que se centrarán en el desarrollo del vocabulario, estructuras básicas y el uso de las wh-questions, para ello se hará uso de una metodología activa y participativa en base a la comprensión check, repetición y la iniciación de los conocimientos previos denominado feed back.

\section{Metodología}

La presente investigación es cuasi-experimental ya que se centró en la aplicabilidad del manual didáctico que contiene estrategias comunicativas, a fin de desarrollar el lenguaje básico en inglés, en estudiantes no videntes de la asociación APRODVICH, a quienes mediante observación y una lista de cotejo, se les aplicó rúbricas de evaluación para determinar el proceso de aprendizaje con la ejecución de las actividades planificadas para la mejora del desarrollo verbal. 
El trabajo de investigación tuvo un alcance correlacional ya que analiza la relación y correlación de la variable dependiente que es el desarrollo del inglés, con las variables independientes que es la aplicación de estrategias de comunicación a través del manual. Lo que además dio lugar a la comprobar las hipótesis de la investigación.

El proceso de investigación se realizó in situ, esto es con los estudiantes no videntes de la asociación APRODVICH de la ciudad de Riobamba, provincia de Chimborazo. A través de una observación directa se aplicó los ensayos, que permitió determinar los principales problemas de aprendizaje del idioma inglés, y así tomar las medidas necesaria para mejorar es proceso de enseñanza aprendizaje. Además se realizó una investigación bibliográfica con la finalidad de sustentar de manera clara y precisa el fundamento científico y teórico de las dos variables.

Para llevar a cabo la investigación se realizó un seguimiento continuo del desenvolvimiento de los estudiantes en el desarrollo del habla del Inglés de acuerdo a la aplicación del manual didáctico. La base de la investigación se sustenta en la planificación académica (Silabos), el cual fue creado con los contenidos mínimos requeridos para el aprendizaje del idioma, con temas como vocabulario, gramática, estructura y wh-questions.

En segunda fase se aplicó actividades para mejorar el habla del inglés, mismas que fueron evaluadas a través de una rúbrica que determinó el aprendizaje, el entendimiento y la pronunciación. Los datos obtenidos a través de las estrategias comunicativas fueron analizados e interpretados.

Una vez superadas las etapas del vocabulario, gramática y verbo Tobe, se puso énfasis en las wh-question que al igual que las anteriores fueron evaluadas con rúbricas para mejorar el habla del idioma, a través de dos escenarios (pre y post). Los resultados encontrados fueron analizados a través de pruebas estadísticas para contrarrestar el nivel de confianza y aceptar o rechazar la hipótesis planteada en la investigación.

La población en el contexto de la presente investigación es 8 estudiantes de APRODVICH de la ciudad de Riobamba, por ser la población tan reducida se trabajó con todo el universo.

\section{Resultados}

Previo a la implementación del manual se evaluó a los ocho estudiantes no videntes de la Asociación. Para ello se realizó una serie de preguntas sobre la frecuencia de uso de las estrategias metodológicas en el aprendizaje del idioma. En cuando a la compresión, vocabulario, dominio y aprendizaje del idioma ingles estas variables se resumen en el cuadro 2. 
Cuadro 2: Resumen de resultados antes y después de la implementación del manual

\begin{tabular}{|c|c|c|c|c|c|c|c|c|c|}
\hline \multirow[b]{2}{*}{$\begin{array}{l}\text { HIPÓ } \\
\text { TESI } \\
\mathrm{S}\end{array}$} & \multirow[b]{2}{*}{ INDICADORES } & \multicolumn{3}{|c|}{ ANTES } & \multirow{2}{*}{$\begin{array}{c}\text { TOTAL } \\
\text { DE } \\
\text { ESTUD } \\
\text { IANTE } \\
\text { S } \\
\text { EVALU } \\
\text { ADOS } \\
\end{array}$} & \multicolumn{3}{|c|}{ DESPUÉS } & \multirow[b]{2}{*}{$\begin{array}{l}\text { TOTAL } \\
\text { DE } \\
\text { ESTUDIA } \\
\text { NTES } \\
\text { EVALUA } \\
\text { DOS }\end{array}$} \\
\hline & & $\begin{array}{l}\text { SIE } \\
\text { MP } \\
\text { RE }\end{array}$ & $\begin{array}{l}\text { A } \\
\text { VEC } \\
\text { ES }\end{array}$ & $\begin{array}{c}\mathrm{N} \\
\mathrm{U} \\
\mathrm{NC} \\
\mathrm{A}\end{array}$ & & $\begin{array}{l}\text { SIE } \\
\text { MP } \\
\text { RE }\end{array}$ & $\begin{array}{l}\text { A } \\
\text { VEC } \\
\text { ES }\end{array}$ & $\begin{array}{l}\mathrm{NU} \\
\mathrm{NC} \\
\mathrm{A}\end{array}$ & \\
\hline \multirow{7}{*}{ 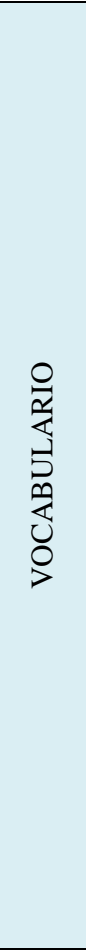 } & $\begin{array}{l}\text { Demuestra mayor } \\
\text { asimilación de } \\
\text { palabras en Inglés }\end{array}$ & 1 & 0 & 7 & 8 & 6 & 1 & 1 & 8 \\
\hline & $\begin{array}{c}\text { Pronuncia las } \\
\text { palabras aprendidas } \\
\text { de manera correcta }\end{array}$ & 0 & 2 & 6 & 8 & 5 & 2 & 1 & 8 \\
\hline & $\begin{array}{l}\text { Identifica las } \\
\text { palabras en inglés } \\
\text { relacionado con } \\
\text { objetos de su } \\
\text { entorno }\end{array}$ & 2 & 1 & 5 & 8 & 7 & 0 & 1 & 8 \\
\hline & $\begin{array}{c}\text { Almacena } \\
\text { progresivamente } \\
\text { nuevas palabras en } \\
\text { su vocabulario } \\
\end{array}$ & 2 & 2 & 4 & 8 & 6 & 2 & 0 & 8 \\
\hline & $\begin{array}{c}\text { Aplica las nuevas } \\
\text { palabras de inglés } \\
\text { en la comunicación } \\
\text { con sus } \\
\text { compañeros }\end{array}$ & 2 & 3 & 3 & 8 & 5 & 2 & 1 & 8 \\
\hline & $\begin{array}{c}\text { Expresa de manera } \\
\text { correcta las } \\
\text { palabras conocidas }\end{array}$ & 5 & 2 & 1 & 8 & 8 & 0 & 0 & 8 \\
\hline & $\begin{array}{l}\text { Repite palabras en } \\
\text { inglés expresadas } \\
\text { por sus compañeros }\end{array}$ & 4 & 3 & 1 & 8 & 5 & 2 & 1 & 8 \\
\hline \multirow{7}{*}{ 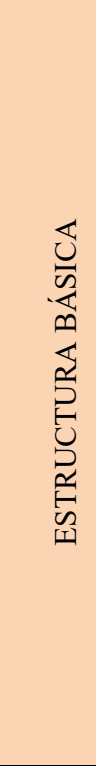 } & $\begin{array}{c}\text { Expresa oraciones } \\
\text { en inglés repitiendo } \\
\text { lo que escucha }\end{array}$ & 0 & 1 & 7 & 8 & 4 & 3 & 1 & 8 \\
\hline & $\begin{array}{c}\text { Identifica las } \\
\text { oraciones en } \\
\text { presente, pasado y } \\
\text { futuro }\end{array}$ & 0 & 2 & 6 & 8 & 5 & 2 & 1 & 8 \\
\hline & $\begin{array}{l}\text { Utiliza el verbo to } \\
\text { be en oraciones }\end{array}$ & 0 & 0 & 8 & 8 & 6 & 1 & 1 & 8 \\
\hline & $\begin{array}{l}\text { Comprende la } \\
\text { oraciones } \\
\text { estructuradas } \\
\end{array}$ & 2 & 2 & 4 & 8 & 7 & 1 & 0 & 8 \\
\hline & $\begin{array}{c}\text { Traduce a español } \\
\text { las oraciones } \\
\text { escuchadas }\end{array}$ & 0 & 1 & 7 & 8 & 6 & 1 & 1 & 8 \\
\hline & $\begin{array}{c}\text { Utiliza el verbo to } \\
\text { be relacionando } \\
\text { con las palabras } \\
\text { aprendidas } \\
\end{array}$ & 3 & 2 & 3 & 8 & 6 & 2 & 0 & 8 \\
\hline & Interrelaciona sus & 1 & 2 & 5 & 8 & 7 & 1 & 0 & 8 \\
\hline
\end{tabular}




\begin{tabular}{|c|c|c|c|c|c|c|c|c|c|}
\hline & $\begin{array}{l}\text { conocimientos con } \\
\text { sus compañeros }\end{array}$ & & & & & & & & \\
\hline \multirow{8}{*}{ 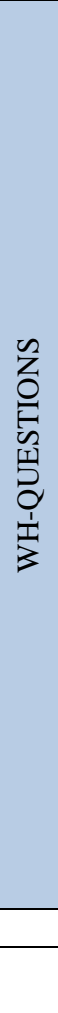 } & $\begin{array}{c}\text { Responde a las } \\
\text { preguntas } \\
\text { utilizando los } \\
\text { verbos to-be }\end{array}$ & 0 & 0 & 8 & 8 & 5 & 2 & 1 & 8 \\
\hline & $\begin{array}{c}\text { Complementa } \\
\text { oraciones según las } \\
\text { preguntas } \\
\text { realizadas por el } \\
\text { docente } \\
\end{array}$ & 1 & 3 & 4 & 8 & 5 & 3 & 0 & 8 \\
\hline & $\begin{array}{c}\text { Identifica la } \\
\text { estructura de las } \\
\text { preguntas } \\
\text { realizadas por el } \\
\text { docente }\end{array}$ & 2 & 1 & 5 & 8 & 4 & 3 & 1 & 8 \\
\hline & $\begin{array}{l}\text { Realiza preguntas a } \\
\text { sus compañeros en } \\
\text { inglés }\end{array}$ & 4 & 3 & 1 & 8 & 7 & 1 & 0 & 8 \\
\hline & $\begin{array}{c}\text { Comprende el } \\
\text { mensaje de la } \\
\text { oración según la } \\
\text { pregunta realizada }\end{array}$ & 4 & 2 & 2 & 8 & 8 & 0 & 0 & 8 \\
\hline & $\begin{array}{c}\text { Demuestra } \\
\text { aprendizaje } \\
\text { significativo del } \\
\text { inglés }\end{array}$ & 1 & 3 & 4 & 8 & 6 & 2 & 0 & 8 \\
\hline & TOTAL & 34 & 35 & 91 & \multirow[b]{2}{*}{$100 \%$} & 118 & 31 & 11 & \multirow[b]{2}{*}{$100 \%$} \\
\hline & PORCENTAJE & $21 \%$ & $22 \%$ & $\begin{array}{l}57 \\
\%\end{array}$ & & $74 \%$ & $19 \%$ & $7 \%$ & \\
\hline
\end{tabular}

Realizado por: Guadalupe Luis, 2015

La siguiente figura resume los resultados de la tabla.

Figura 1: Evolución antes y después de la aplicación del manual

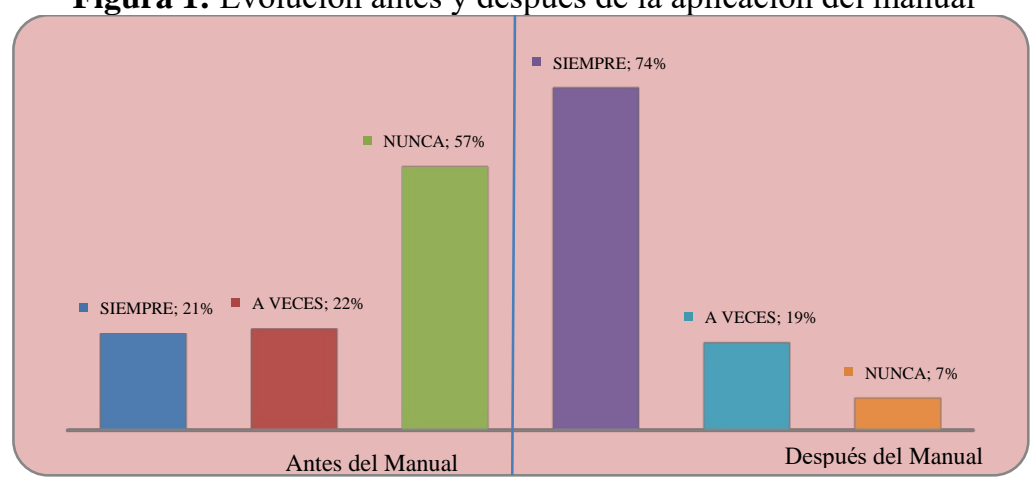

Realizado por: Guadalupe Luis, 2015

La figura 1. Muestra que los estudiantes antes de la aplicación del manual utilizaban siempre estrategias metodológicas en un $21 \%$, en tanto que el $22 \%$ a veces y el $57 \%$ nunca. Después del desarrollo del proceso enseñanza aprendizaje con la utilización del manual los resultados variaron 
siendo ahora un 74\% el número de estudiantes que siempre desarrollan el habla del inglés con el uso de estrategias metodológicas, el 19\% a veces y el $7 \%$ nunca.

De acuerdo a los resultados obtenidos se puede evidenciar que los estudiantes no videntes de la Asociación APRODVICH tienen dificultades en el área de Inglés, precisamente porque no venían utilizando de manera adecuada estrategias comunicativas, ante lo cual fue importante orientar a los docentes acerca de la importancia que tiene en el proceso de enseñanzaaprendizaje a través del vocabulario, la orientación en base a las estructuras básicas y el uso de cuestionarios, así como otras estrategias comunicacionales establecidas en el manual. Los docentes pudieron evidenciar la diferencia significativa en el aprendizaje del idioma al aplicar el manual y la recepción y motivación de los estudiantes por mejorar sus conocimientos en el idioma.

\section{Demostración de la hipótesis $\mathbf{N}^{0} 1$}

H1: La elaboración y aplicación de un manual didáctico con estrategias comunicativas a través del vocabulario desarrolla el habla del inglés básico en los estudiantes no videntes de la asociación APROVICH, ciudad de Riobamba, provincia de Chimborazo.

Figura 2: Zona de aceptación de la Hipótesis Específica $\mathrm{N}^{\circ} 1$

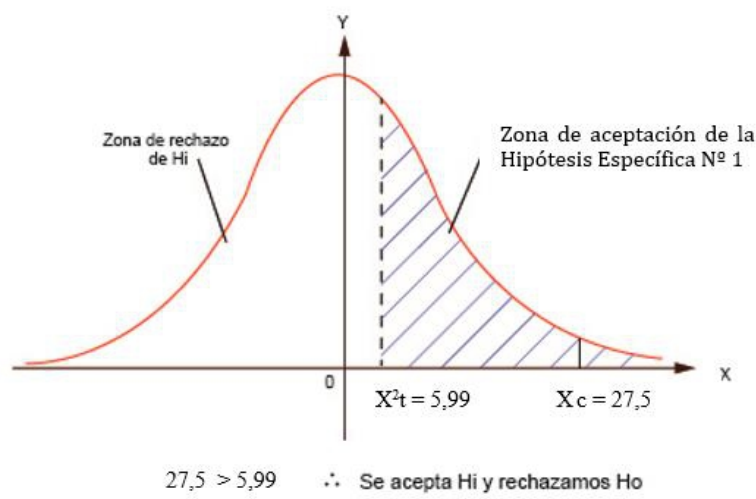

Realizado por: Guadalupe Luis, 2015

\section{Decisión}

El valor de $X^{2}$ cal $=27,5$; con 2 grados de libertad y un nivel de 0,05 de nivel significancia se obtiene el $X^{2}$ tab de 5,99 y que se encuentra en la zona de rechazo de Ho. Esto indica que se rechaza la hipótesis nula (Ho) y se acepta la hipótesis de investigación 1, es decir que: La elaboración y aplicación de un manual con estrategias comunicativas a través del vocabulario desarrolla la habilidad de hablar inglés básico en los estudiantes no videntes de la asociación APRODVICH. 


\section{Demostración de la hipótesis $\mathrm{N}^{0} 2$}

H2: La elaboración y aplicación de un manual con estrategias comunicativas a través de las estructuras básicas desarrolla la habilidad de hablar inglés básico en los estudiantes no videntes de la asociación APROVICH.

Figura 3: Zona de aceptación de la Hipótesis Específica $\mathrm{N}^{\mathrm{o}} 2$

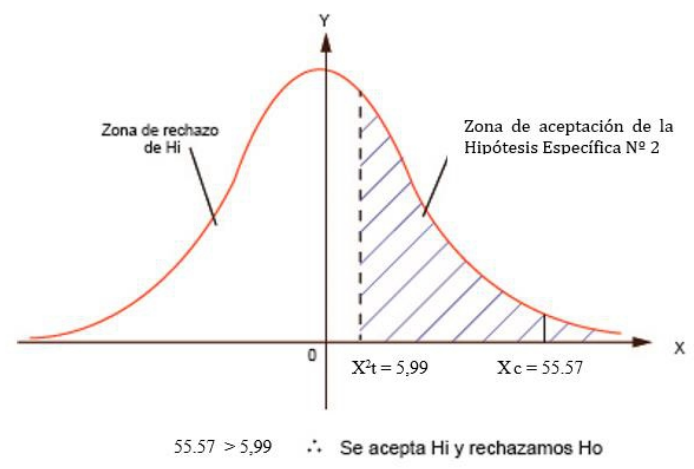

Realizador por: Guadalupe Luis, 2015

\section{Decisión}

El valor de $X^{2}$ cal $=55.57$; con 2 grados de libertad y un nivel de 0,05 de nivel significancia se obtiene el $\mathrm{X}^{2}$ tab de 5,99 y que se encuentra en la zona de rechazo de Ho. Esto indica que se rechaza la hipótesis nula (Ho) y se acepta la hipótesis de investigación 1, es decir que: La elaboración y aplicación de un manual con estrategias comunicativas a través de basic structures desarrolla el habla del inglés básico en los estudiantes no videntes de la asociación APRODVICH.

\section{Demostración de la hipótesis $\mathbf{N}^{0} 3$}

H3: La elaboración y aplicación de un manual con estrategias comunicativas a través del uso de las wh-questions basadas en inglés básico desarrolla el habla de los estudiantes no videntes de la asociación APROVICH.

Gráfico 04: Zona de aceptación de la Hipótesis Específica № 3

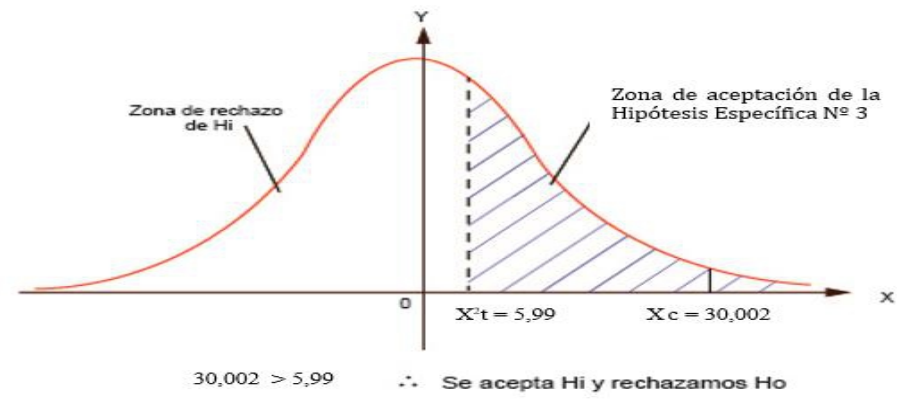

Realizado por: Guadalupe Luis, 2015 


\section{Decisión}

El valor de $\mathrm{X}^{2}$ cal $=30,002$; con 2 grados de libertad y un nivel de 0,05 de nivel significancia se obtiene el $\mathrm{X}^{2}$ tab de 5,99 y que se encuentra en la zona de rechazo de Ho. Esto indica que se rechaza la hipótesis nula (Ho) y se acepta la hipótesis de investigación 1, es decir que: La elaboración y aplicación de un manual con estrategias comunicativas a través del uso de las whquestions basadas en Inglés básico desarrolla el habla en los estudiantes no videntes de la asociación APRODVICH.

\section{Decisión de la hipótesis general}

Se realizó la comprobación de las 3 hipótesis específicas: en la primera el valor de $X^{2}$ cal $=27.5$ es mayor que $X^{2}$ tab $=5.99$, la segunda hipótesis el $\mathrm{X}^{2}$ cal $=55.57$ es mayor que $\mathrm{X}^{2} \mathrm{tab}=5.99$ y la tercera hipótesis el $\mathrm{X}^{2}$ cal $=30.002$ es mayor que $\mathrm{X}^{2}$ tab $=5.99$ es decir que se rechazan las hipótesis nulas Ho y se aceptan las 3 hipótesis de investigación, comprobando la hipótesis general que dice: La elaboración y aplicación de un manual con estrategias comunicativas desarrolla el habla del Inglés básico de los estudiantes no videntes de la asociación APRODVICH, ciudad de Riobamba, provincia de Chimborazo, durante el año lectivo 2014-2015.

\section{Conclusion}

- La aplicación del manual con estrategias comunicativas permitió a los estudiantes no videntes encaminar el aprendizaje del idioma inglés básico a través de la ejercitación del vocabulario, a través de ejercicios de repetición para mejorar la pronunciación de las palabras.

- Las actividades relacionadas con el manual, sirvió para ir enlazando los conocimiento adquiridos durante el proceso enseñanza aprendizaje. El uso del verbo To be, permite una mayor interacción entre los estudiantes obligándolos a hablar más para entenderse.

- La utilización de las estrategias comunicativas para la formulación de preguntas interactivas utilizando wh-questions, no solo generó más confianza en los estudiantes, sino permitió además una mayor interacción entre estudiante y estudiante; Docente y estudiante, observando un aprendizaje significativo del idioma.

- Las pruebas estadísticas permitieron confirmar la ventaja de estudiar inglés, con el uso del manual didáctico con estrategias comunicativas, los estudiantes no videntes lograron eliminaron las barreras y obtuvieron un aprendizaje significativo del idioma.

\section{References:}

1. Aja Fernández, J. (2012). Manual de la Educación. Barcelona: Océano Grupo Editorial, S.A. 
2. Bandura, E. (2003). El Lenguaje Oral en los Niños. Madrid: SIMA.

3. Bernard, M., \& Risle, M. (1990). Manual de Orientación Vocacional. Tomo 1. Chile.

4. Martínez Bonafé, J. (2002). Siete cuestiones y una propuesta. Cuadernos de pedagogía. México: Trillas.

5. Bonafé, J. M. (2002). Políticas del libro de texto escolar. Ediciones Morata

6. Castañeda Yánez, M. (2002). Los medios de comunicación y la tecnología educativa. . México: Trillas.

7. Castañeda, M. (2009). El proceso de aprendizaje. Segunda edición. México: Trillas.

8. Cultural, E. (2007). Técnicas de estudio . Madrid-España: Cultural S.A.

9. Deuchar, M. (2007). Ensayo del lenguaje de sordos y el proceso gramatical. New York: Falmer.

10. Escudero Yerena, T. (2008). La comunicación en la enseñanza. México: Trillas.

11. Fonseca, M. (2006). Imagen y enseñanza. Serie nuevos métodos de enseñanza. México: UNAM.

12. Gispert, C. (2006). Manual de la educación. Barcelona-España: Grupo Editorial Océano. S.A.

13. Ministerio de Educación. (2010). Actualización y Fortalecimiento Curricular de Educación Básica. Quito.

14. Owens, J., \& E., R. (1999). Language development: An introduction. Boston: Allyn and Bacon.

15. Perrenoud, P. (1999). Formación de formadores. Ginebra.

16. Rivers, W. (2007). Estrategias de aprendizaje del inglés. Washington: Adventure Works.

17. Urdanigo, E., \& Donoso, M. (2010). Enseñanza del idioma Inglés. Jipijapa.

18. Zavalloni, R. (2011). Introducción a la pedagogía especial. Barcelona: Cultural S.A. 